\title{
IncRNA PVT1 promotes the angiogenesis of vascular endothelial cell by targeting miR-26b to activate CTGF/ANGPT2
}

\author{
JIFU ZHENG ${ }^{1,2^{*}}$, LILI HU ${ }^{1,3^{*}}$, JING CHENG $^{2}$, JING XU $^{2}$, ZHIQIANG ZHONG ${ }^{2}$, \\ YUAN YANG $^{2}$ and ZHENG YUAN ${ }^{2}$ \\ Departments of ${ }^{1}$ Cardiovascular Medicine, ${ }^{2}$ Hematology and ${ }^{3}$ Nephrology, \\ The Second Affiliated Hospital of Nanchang University, Nanchang, Jiangxi 330006, P.R. China
}

Received September 12, 2017; Accepted March 22, 2018

DOI: 10.3892/ijmm.2018.3595

\begin{abstract}
Angiogenesis is essential for various biological processes, including tumor blood supply delivery, cancer cell growth, invasion and metastasis. Plasmacytoma variant translocation 1 (PVT1) long noncoding RNA (lncRNA) has been previously reported to affect angiogenesis of glioma microvascular endothelial cells by regulating microRNA (miR)-186 expression level. However, the specific underlying molecular mechanism of PVT1 regulation of angiogenesis in vascular endothelial cells remains to be elucidated. The present study investigated the role of PVT1 in cell proliferation, migration and vascular tube formation of human umbilical vein endothelial cells (HUVECs) using MTT assay, Transwell migration assay and in vitro vascular tube formation assay, respectively. In order to determine the effect of miR-26b on cell proliferation, migration and vascular tube formation of HUVECs, miR-26 mimic or miR-26b inhibitor were transfected into HUVECs. Reverse transcription-quantitative polymerase chain reaction and western blotting were conducted to quantify the mRNA and protein expression levels of target genes. The present study confirmed that miR-26b bound 3'-untranslated region (3'-UTR) and subsequently influenced gene expression level using dual luciferase reporter assay. The current study observed that PVT1 affected cell proliferation, migration and in vitro vascular tube formation of HUVECs. In addition, it was determined that PVT1 was able to bind and degrade miR-26b to promote connective tissue growth factor (CTGF) and angiopoietin 2 (ANGPT2) expression. miR-26b was also identified to have a suppressive role in cell proliferation,
\end{abstract}

Correspondence to: Dr Lili Hu, Department of Cardiovascular Medicine and Nephrology, The Second Affiliated Hospital of Nanchang University, 1 Minde Road, Nanchang, Jiangxi 330006, P.R. China

E-mail: hulili_2017@163.com

*Contributed equally

Key words: angiogenesis, vascular endothelial cell, microRNA-26b, connective tissue growth factor, angiopoietin 2 migration and in vitro vascular tube formation of HUVECs via binding 3'-UTR regions and downregulating CTGF and ANGPT2 expression levels. The current findings may improve the understanding of the underlying mechanism of PVT1 contributing to angiogenesis of vascular endothelial cells and offer rationale for targeting PVT1 to treat angiogenesis dysfunction-associated diseases, including cancer metastasis.

\section{Introduction}

A previous study suggested that various biological processes are determined by the regulatory potential of the noncoding portions of the genome. It has been previously estimated that $\sim 1.5 \%$ of the genome is responsible for protein coding, whereas a number of noncoding regulatory elements are transcribed into noncoding RNA (ncRNA) (1). Long noncoding (lncRNAs) (>200 nucleotides) are a novel form of ncRNAs, which have been identified to exert their gene transcription regulatory effect through the epigenetic regulatory mechanism. LncRNA plasmacytoma variant translocation 1 (PVT1) has been reported to be associated with cell proliferation, invasion and metastasis, apoptosis and tumor prognosis (2-7). Although, some previous studies have revealed that lncRNAs such as MEG3 maternally expressed 3, HOX transcript antisense RNA had an important role in angiogenesis by directly regulating vascular endothelial growth factor (VEGF) and angiopoietin 2 (ANGPT2); however, whether PVT1 had a similar function remains to be elucidated (8-10). A recent study by Ma et al (11) observed that PVT1 regulated growth, migration and angiogenesis of glioma microvascular endothelial cells by targeting microRNA (miR)-186; therefore, it should be considered if other pathways or downstream targets mediate PVT1 regulation of angiogenesis in vascular endothelial cells.

MicroRNA is a class of 22 nucleotide noncoding RNAs. They may regulate gene expression through recognizing and binding to the 3'-untranslated region (3'-UTR) of target gene mRNAs, leading to mRNA degradation or translational suppression (12). It has been estimated that approximately one out of three human genes are regulated by miRNAs $(13,14)$. Previous studies have revealed that miRNAs have a critical role in a variety of cellular processes in healthy and ailing individuals, including pro-angiogenic therapeutics to reconstruct vasculature for patients with ischemic heart and 
peripheral vascular diseases (15-18). A previous study reported that miR-26a regulates pathological and physiological angiogenesis by targeting its downstream bone morphogenetic protein/SMAD family member 1 signaling (19). Another study demonstrated that lncRNA PVT1 is a miR-26b sponge and promoted melanoma progression, which indicated that lncRNA PVT1 may regulate angiogenesis via interaction with miR-26b (20).

Connective tissue growth factor (CTGF) is a member of the $\mathrm{CCN}$ family which consists of cysteine-rich proteins. It has been previously demonstrated that CTGF is associated with fibrosis, tissue remodeling and tumorigenesis (21). It is of note that CTGF has a promoter role in the regulation of vessel growth during development, wound healing and vascular disease, suggesting CTGF is an angiogenetic inducer (22). In previous studies, CTGF has been revealed to be a direct downstream target of miR-26b, indicating that miR-26b may influence angiogenesis via downregulation of CTGF gene expression (20).

The present study revealed that PVT1 directly interacts with miR-26b to reduce the expression level, which subsequently promoted CTGF and ANGPT2 expression levels, which contributed to cell proliferation, migration and angiogenesis of vascular endothelial cells.

\section{Materials and methods}

Cell line. Human umbilical vein endothelial cells (HUVECs) and 293T cells were purchased from America Type Culture Collection (Manassas, VA, USA). HUVECs were cultured in vascular cell basal medium (ScienCell Research Laboratories, Inc., San Diego, CA, USA) supplemented with $10 \mathrm{ng} / \mathrm{ml}$ VEGF at $37^{\circ} \mathrm{C}, 5 \% \mathrm{CO}_{2} .293 \mathrm{~T}$ cells were cultured in Dulbecco's modified Eagle's medium (DMEM) (HyClone; GE Healthcare, Logan, UT, USA) supplemented with $10 \%$ fetal bovine serum $(\mathrm{FBS})(\mathrm{BI})$ and $1 \%$ penicillin $(100 \mathrm{U} / \mathrm{ml}) /$ streptomycin (100 U/ml) (Invitrogen; Thermo Fisher Scientific, Inc., Waltham, MA, USA).

Plasmid transfection and lentivirus package. PVT1 full-length cDNA was cloned into a pEX2 plasmid and the short hairpin RNA (shRNA) targeting PVT1 was cloned into a pLKO.1-TRC vector (all from Shanghai GenePharma Co., Ltd., Shanghai, China), where the transcription was under the control of the U6 promoter. shPVT1 target sequence was 5'-CAGCCATCA TGATGGTACT-3'. In order to generate lentiviruses, the transducing vectors (pPAX2 and pVSVG; Shanghai GenePharma Co., Ltd.) were co-transfected into 293T cells with polybrene (Sigma-Aldrich; Merck Millipore, Darmstadt, Germany). The supernatant was harvested at 24 and $48 \mathrm{~h}$ after transfection, filtered through $0.45 \mu \mathrm{m}$ membrane and concentrated using a centrifugal filter (EMD Millipore, Billerica, MA, USA).

Luciferase reporter assay. The CTGF mutant 3'-UTR was generated by replacing the seed regions of the miR-26b binding sites with 5'-TTGGTT-3' and PVT1 mutant was generated using site-directed mutagenesis (23). Subsequently, the mutant sequence was cloned into the firefly luciferase-expressing vector pGL3 (Shanghai GenePharma Co., Ltd.). As for luciferase assay, the HUVECs were seeded in 24-well plates at $4 \times 10^{4}$ cells/well the day before transfection and transfected with the CTGF wild type or mutant 3'-UTR reporter vector (Shanghai GenePharma Co., Ltd.), PVT1 or PVT1 mutant using Lipofectamine ${ }^{\circledR} 2000$ (Invitrogen; Thermo Fisher Scientific, Inc.). The cells were harvested and lysed $48 \mathrm{~h}$ after transfection and the luciferase activity was assayed using the Dual-Luciferase Reporter system (Promega Corporation, Madison, WI, USA). The $\beta$-lactamase gene of the pGL3 luciferase vector was used for the normalization of the luminescence levels. Three independent experiments were performed.

Transwell migration assay. The cells were transfected with miR-26b mimic using Lipofectamine ${ }^{\circledR} 2000$, miR-26b inhibitor (both from Shanghai GenePharma Co., Ltd.), PVT1 or shPVT1. After $24 \mathrm{~h}$, the cells were starved in medium without serum for another $12 \mathrm{~h}$ and then digested with trypsin and then $3 \times 10^{4}$ cells were seeded in the top chamber of 24-well Transwell culture inserts (Promega Corporation). The medium supplemented with $20 \%$ FBS used as chemoattractant was added to bottom chamber. After $24 \mathrm{~h}$ incubation, the cells were fixed for 10 min with $4 \%$ paraformalin and cells which had not migrated were removed. The cells on the lower side of the filter were stained with $0.005 \%$ crystal violet for $30 \mathrm{~min}$ in $25^{\circ} \mathrm{C}$ and then the number of cells was counted and photographed with an inverted microscope at magnification of x100 and three fields of view.

Cell proliferation assay. The proliferation of HUVECs from various groups were examined via MTT assay. The cells were counted and plated into 96 -well plates at a density of $2 \times 10^{3}$ cells/well for $24 \mathrm{~h}$, then $0.1 \mathrm{mg} / \mathrm{ml} \mathrm{MTT}$ was added to cells at $37^{\circ} \mathrm{C}$ for $3 \mathrm{~h}$ and lysed in DMSO at room temperature for $30 \mathrm{~min}$. Finally, absorbance was quantified at $490 \mathrm{~nm}$ using a microplate reader (Omega Bio-Tek, Inc., Norcross, GA, USA).

In vitro vascular tube formation assay. To examine the ability of endothelial tube formation in vitro, the present study used 15-well $\mu$-slides (ibidi GmbH, Martinsried, Germany) coated with $10 \mu \mathrm{l}$ Matrigel as previously described (23). To determine the effect of PVT1 and miR-26b on tube formation, HUVECs were pretreated with $\mathrm{CoCl}_{2}$ and transfected with PVT1 and miR-26b mimics or siRNAs against PVT1 or miR-26b. The cells were grown in Medium DMEM (ScienCell Research Laboratories, Inc., San Diego, CA, USA) supplemented 10\% FBS (Thermo Fisher Scientific, Inc.) and $3.75 \mu \mathrm{g} / \mathrm{ml}$ endothelial cell growth supplement (BD Biosciences, New Jersey, USA). The tube length was measured using MetaMorph version 7.8.10 (Molecular Devices, LLC, Sunnyvale, CA, USA) and compared with the control. The micrographs were captured and processed with an inverted microscope.

Reverse transcription-quantitative polymerase chain reaction (RT-qPCR). Total RNA was extracted using TRIzol method. The cells were lysed with TRIzol buffer (Thermo Fisher Scientific, Inc.), and $200 \mu \mathrm{l}$ chloroform was added to mixture. The resulting solution was centrifuged at $10,000 \mathrm{x} \mathrm{g}$ for $10 \mathrm{~min}$ at $4^{\circ} \mathrm{C}$. The supernatant was harvested and mixed with equivalent volume of isopropanol. The resultant was subjected to 
centrifugation at $10,000 \mathrm{x}$ for $10 \mathrm{~min}$ at $4^{\circ} \mathrm{C}$. The supernatant was removed and $75 \%$ ethanol was added to wash the pellet and centrifuged at 7,500 $\mathrm{xg}$ for $5 \mathrm{~min}$ at $4^{\circ} \mathrm{C}$. The ethanol was discarded and the pellet dried, 20-30 $\mu$ l RNAse-free $\mathrm{H}_{2} \mathrm{O}$ was used to elute the RNA pellet.

Subsequently, $1 \mu \mathrm{g}$ total RNA underwent reverse transcription using PrimeScript kit according to manufacturer's protocol (Takara Biotechnology Co., Ltd., Dalian, China). For qPCR, this experiment was performed using SYBR (Guangzhou RiboBio Co., Ltd., Guangzhou, China) as probe dye and detected the signal by the standard protocol. The expression of miR-26b was detected using a Bulge-Loop ${ }^{\mathrm{TM}}$ miRNA qRT-PCR Primer set (Guangzhou RiboBio Co., Ltd.) according to the manufacturer's protocol. cDNA was synthesized from total RNA using the PrimeScript kit at $25^{\circ} \mathrm{C}$ for $10 \mathrm{~min}$, at $42^{\circ} \mathrm{C}$ for $50 \mathrm{~min}$, at $95^{\circ} \mathrm{C}$ for $5 \mathrm{~min}$. cDNA was then amplified following cycling conditions: One initial PCR activation step at $95^{\circ} \mathrm{C}$ for $15 \mathrm{~min}$ followed by 40 cycles of denaturation at $94^{\circ} \mathrm{C}$ for $15 \mathrm{sec}$, annealing at $53^{\circ} \mathrm{C}$ for $30 \mathrm{sec}$, and elongation at $72^{\circ} \mathrm{C}$ for $30 \mathrm{sec}$. The U6 and GAPDH were used as internal control. The following primers were used: PVT1 forward (F), 5'-GGGGAATAACGCTGGTGGAA-3' and reverse (R), 5'-CCCATGGACATCCAAGCTGT-3'; CTGF F, 5'-GAGAGTCCTTCCAGAGCAGC-3' and R, 5'-CATAGT TGGGTCTGGGCCAA-3'; ANGPT2 F, 5'-CCCTACGTG TCCAATGCTGT-3' and R, 5'-CCGCTGTTTGGTTCAACA GG-3'; U6 F, 5'-CTCGCTTCGGCAGCACATATACTA-3' and U6 R, 5'-ACGAATTTGCGTGTCATCCTTGCG-3'; GAPDH F, 5'-GAGTCAACGGATTTGGTCGT-3' and R, 5'-TTGATT TTGGAGGGATCTCG-3'. Cq values were used for quantification using a previously described protocol (24). cDNA was prepared for three times and RT-qPCR was repeated in triplicate parallel experiments.

Western blotting. Cells were harvested and washed with PBS. Subsequently SDS loading buffer (Sigma-Aldrich; Merck Millipore) was used to lyse cells. The lysates were boiled at $95^{\circ} \mathrm{C}$ for $10 \mathrm{~min}$ and then subjected to centrifugation at $10,000 \mathrm{x} \mathrm{g}$ for $1 \mathrm{~min}$ at $4^{\circ} \mathrm{C}$. Total protein $(50 \mu \mathrm{g})$ was loaded onto $10 \%$ SDS-PAGE gel and resolved at $120 \mathrm{~V}$ for $30 \mathrm{~min}$ to $1 \mathrm{~h}$. Subsequently, the proteins in the gel were transferred onto a PVDF membrane at $300 \mathrm{~mA}$ for $2-3 \mathrm{~h}$. The membrane was blocked with $5 \%$ non-fat milk in TBST with $0.1 \%$ Tween-20 for $1 \mathrm{~h}$ at room temperature, and then the membrane was incubated with the following primary antibodies at $4{ }^{\circ} \mathrm{C}$ overnight: CTGF (cat no. ab6992; 1:1,000), ANGPT2 (cat no. ab8452; 1:1,000) (both from Abcam, Cambridge, UK), $\beta$-actin (cat no. 60008-1-Ig; 1:5,000; ProteinTech Group, Inc., Chicago, IL, USA). The following day, the membrane was washed with TBST 3 times and incubated with a horseradish peroxidase-conjugated goat anti-rabbit immunoglobulin $\mathrm{G}$ (cat. no. A0208; 1:5,000; Beyotime Institute of Biotechnology, Haimen, China) at room temperature for $1 \mathrm{~h}$. Finally, the membrane was incubated with enhanced chemiluminescent reagent (7Sea Biotech, Shanghai, China) and then exposed using Bio-Rad ChemiDoc Touch Imaging system (Bio-Rad Laboratories, Inc., Hercules, CA, USA).

Statistical analysis. Each experiment was performed three times. All data are presented as mean \pm standard deviation.
Comparisons of parameters were performed using a two-tailed unpaired Student's t-test using Prism version 6.0 (GraphPad Software, Inc., La Jolla, CA, USA). $\mathrm{P}<0.05$ was considered to indicate a statistically significant difference.

\section{Results}

miR-26b inhibits cell proliferation, migration and in vitro tube formation in HUVECs. In order to investigate the role of miR-26b in angiogenesis of vascular endothelial cells, and miR-26b-overexpressing HUVECs were constructed by transfection with miR-26b mimic. miR-26b-depleted HUVECs were established by transfecting the cells with an miR-26b inhibitor. RT-qPCR revealed that miR-26b expression was elevated in cells transfected with miR-26b mimic compared to mimic negative control (NC), whereas miR-26b level was attenuated following transfection with miR-26b inhibitor relative to negative control (Fig. 1A).

The present study identified that miR-26b inhibited the migration of HUVECs in Transwell assays and inhibition miR-26b promoted this migration (Fig. 1B). Subsequently, the present study investigated whether miR-26b affected proliferation of HUVECs. The MTT assay demonstrated that cell proliferation of cells overexpressing mir-26 was significantly reduced, conversely depletion of miR-26b increased the cell proliferation ability of HUVECs (Fig. 1C). In vitro a vascular tube formation assay revealed that miR-26b overexpression reduced the length of the vascular tubes; however, miR-26b depletion led to the formation of longer vascular tubes, which was analyzed statistically by branching points formed by various groups of HUVECs (Fig. 1D). These findings indicated that miR-26b has a suppressive role in cell proliferation, migration and tube formation in HUVECs.

miR-26b suppresses expression of PVT1 and CTGF. In order to determine the molecular mechanism by which miR-26b contributed to cell proliferation, migration and tube formation in HUVECs, the present study focused on the expression level of previously reported genes, CTGF and ANGPT2. Using RT-qPCR it was observed that compared with the mimic NC group, miR-26b overexpression led to an attenuated expression level of CTGF and ANGPT2 (Fig. 2A and B) and unexpectedly of PVT1 (Fig. 2C). Conversely, miR-26b inhibition increased their expression levels. Additionally, compared with the control (mimic NC), protein expression levels of CTGF and ANGPT2 were reduced in the group overexpressing miR-26b. In agreement with the RT-qPCR findings, miR-26b loss led to upregulation of CTGF and ANGPT2 protein expression levels (Fig. 2D). Collectively, the current findings suggested that miR-26b has a suppressive role in the angiogenesis of HUVECs primarily via downregulation of CTGF and ANGPT2.

miR-26b directly interacts with 3'-UTR of CTGF. As microRNAs exert their biological effects through binding the cognate target sequence of mRNAs to block translation or lead to degradation of mRNAs, the current study aimed to clarify whether miR-26b inhibited CTGF expression by directly binding to CTGF mRNA, which then led to accelerated degradation. Bioinformatics analysis using www.microrna. 

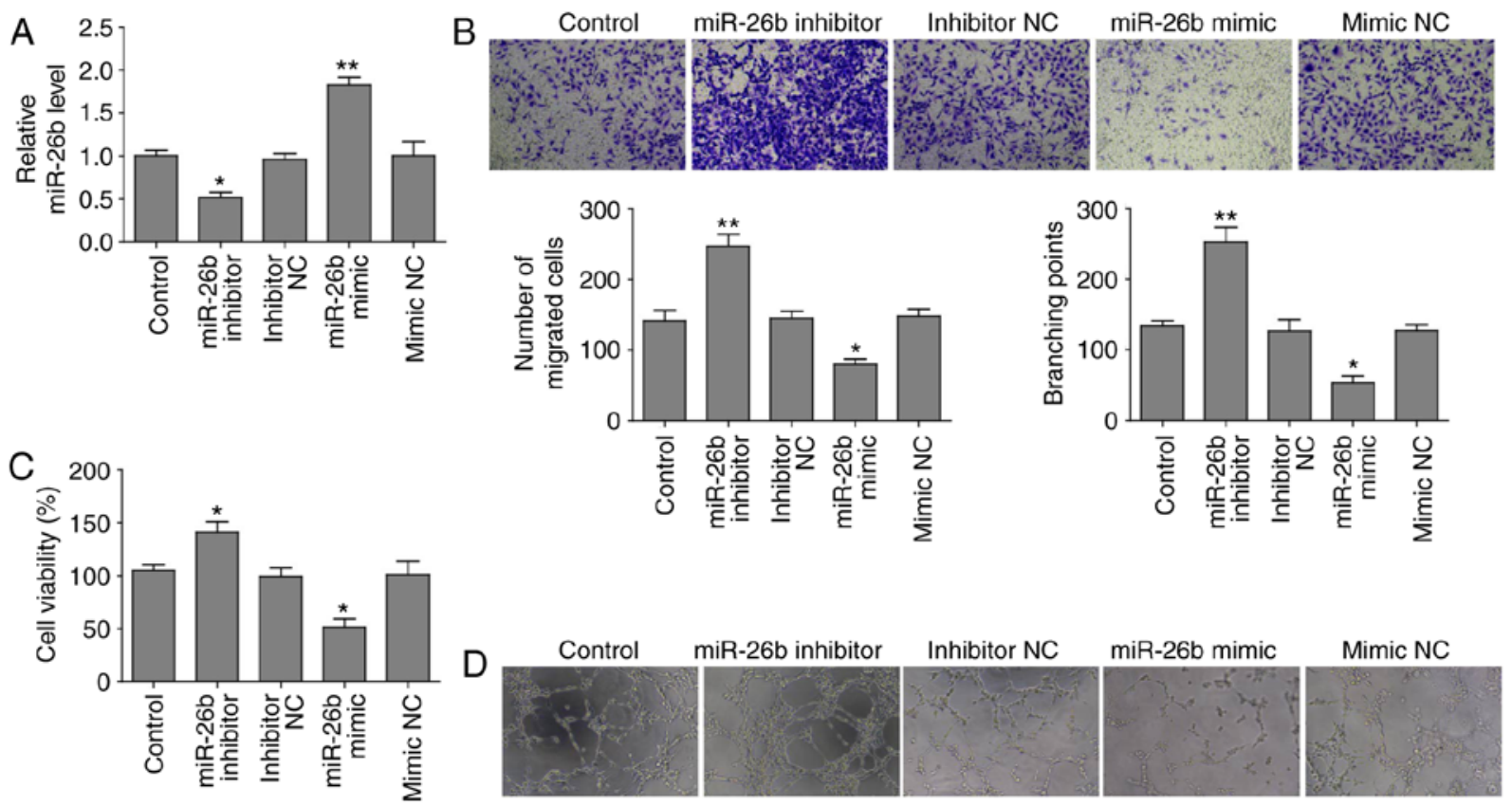

Figure 1. miR-26b inhibits cell proliferation, migration and in vitro tube formation of HUVECs. (A) Reverse transcription-quantitative polymerase chain reaction analysis of miR-26b expression levels in HUVECs transfected with the indicated plasmids. U6 was used as internal control. ${ }^{* *} \mathrm{P}<0.01,{ }^{*} \mathrm{P}<0.05$ vs. respective NC groups. (B) Transwell migration assay of HUVECs cells transfected with the indicated plasmids (upper) and statistical analysis of the migrated cell number ${ }^{* *} \mathrm{P}<0.01,{ }^{*} \mathrm{P}<0.05$ vs. respective NC groups. (C) Viability assay of HUVECs cells transfected with the indicated plasmids by MTT assay. ${ }^{*} \mathrm{P}<0.05$. (D) In vitro tube formation assay of HUVECs cells transfected with the indicated plasmids (upper) and statistical analysis of the tubule junctions. ** $\mathrm{P}<0.01$, ${ }^{*} \mathrm{P}<0.05$ vs. respective NC groups. All data are presented as the mean \pm standard deviation. HUVEC, human umbilical vein endothelial cells; miR, microRNA; $\mathrm{NC}$, negative control.
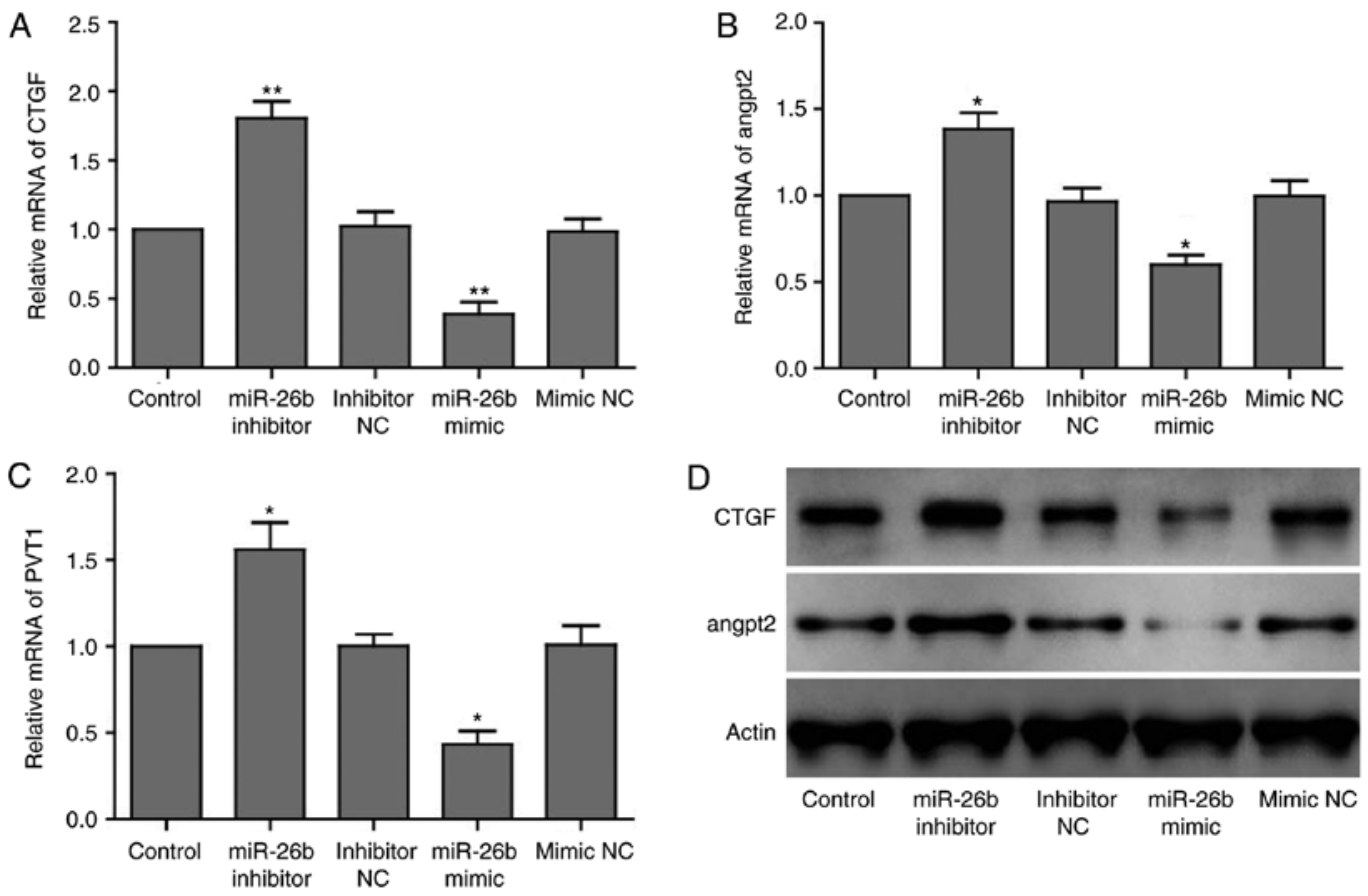

Figure 2. miR-26b suppresses expression of PVT1, CTGF and ANGPT2. (A) Reverse transcription-quantitative polymerase chain reaction analysis of (A) CTGF, (B) ANGPT2 and (C) PVT1 mRNA expression levels in HUVECs transfected with negative controls, miR-26b mimics or inhibitors. GAPDH was used as internal control. Data are presented as the mean \pm standard deviation. $\mathrm{P}<0.05,{ }^{* *} \mathrm{P}<0.01$ vs. respective NC groups. (D) Western blot analysis of CTGF and ANGPT2 protein expression levels in HUVECs transfected with negative controls, miR-26b mimic or inhibitor. $\beta$-actin was used as internal control. HUVEC, human umbilical vein endothelial cells; miR, microRNA; NC, negative control; PVT1, plasmacytoma variant translocation 1; CTGF, connective tissue growth factor; ANGPT2, angiopoietin 2.

org revealed that the $3^{\prime}$-UTR of CTGF may be targeted by miR-26b with large extent of sequence complementarity, which was consistent with a previous study (23) (Fig. 3A). Based on the binding sequence, a mutant CTCF 3'-UTR-containing 


\section{A CTGF $3^{\prime}$ UTR WT 5' CUCAUUUAGACUAUAACUUGAAC 3 hsa-miR-26b-5p $3^{\prime}$ UGGAUAGGACUUAAUGAACUU $5^{\prime}$ \\ CTGF 3'UTR MUT $\quad$ 5' CUCAUUUAGACUAUAUGAACUUC $3^{\prime}$}

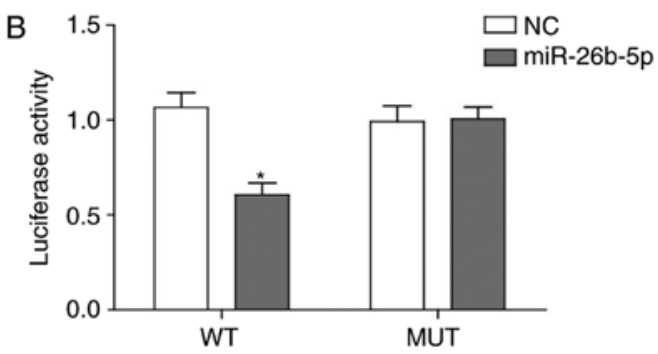

Figure 3. miR-26b directly interacts with 3'-UTR of CTGF. (A) Bioinformatics analysis of match sequence of miR-26b within 3'-UTR of CTGF. MUT CTGF 3'-UTR is the mutation of the match sequence of 3'-UTR of CTGF with miR-26b. (B) Luciferase reporter assay revealed that miR-26b binds to the 3'-UTR of WT CTGF, not MUT CTGF. Relative luciferase activity was quantified and the data were presented as mean \pm standard deviation. "P<0.05 vs. respective NC groups. 3'-UTR, 3'-untranslated region; CTGF, connective tissue growth factor; miR, microRNA; NC, negative control; WT, wild-type; MUT, mutant.

luciferase reported vector and wild-type vector were generated and verified that miR-26b was able to recognize and bind to the wild-type CTGF 3'-UTR to impair activity of luciferase and could not bind to the mutant form of CTGF 3'-UTR (Fig. 3B).

LncRNA PVT1 promotes cell proliferation, migration and in vitro tube formation of HUVECs cell. In order to investigate the biological function of IncRNA PVT1 in vascular endothelial cells, the present study generated PVT1-overexpressing and PVT1-knockdown HUVECs. RT-qPCR revealed that the relative expression of PVT1 in the PVT1 overexpressing cells (pEX2-PVT1) was higher compared with the control cells (pEX2-PVT1-NC). The PVT1 knockdown cells (shPVT1) exhibited substantially reduced PVT1 expression level compared with the negative control (shNC) group cells (Fig. 4A).

Furthermore, it was also observed that PVT1 had a stimulatory role in migration capability of HUVECs. PVT1-overexpressing cells had a higher migration ability compared with the control, whereas shPVT1 cells had decreased migration ability (Fig. 4B). It is of note that PVT1 overexpression markedly promoted cell proliferation of HUVECs, whereas PVT1 depletion impaired cell growth rate of HUVECs (Fig. 4C). Additionally, PVT1 overexpression promoted in vitro vascular tube formation as tube length was increased in pEX2-PVT1 cells compared with shPVT1 cells, which formed fewer tubule junctions (Fig. 4D).

PVT1 suppresses miR-26b and CTGF expression levels. In order to investigate PVT-promoted HUVECs angiogenesis, the present study determined that whether PVT1 promoted angiogenesis through the miR-26b/CTGF axis. Therefore, the present study aimed to determine whether PVT1 affected the expression of miR-26b and its downstream angiogenesis-associated targets. RT-qPCR analysis revealed that PVT1 overexpression led to reduced expression level of miR-26b and increased CTGF and ANGPT2 mRNA expression level. The cells lacking PVT1 following an infection with a PVT1 shRNA-containing virus exhibited elevated level of miR-26b and downregulated mRNA levels of CTGF and ANGPT2 (Fig. 5A-C). In addition, similar changes were observed in terms of the CTGF and
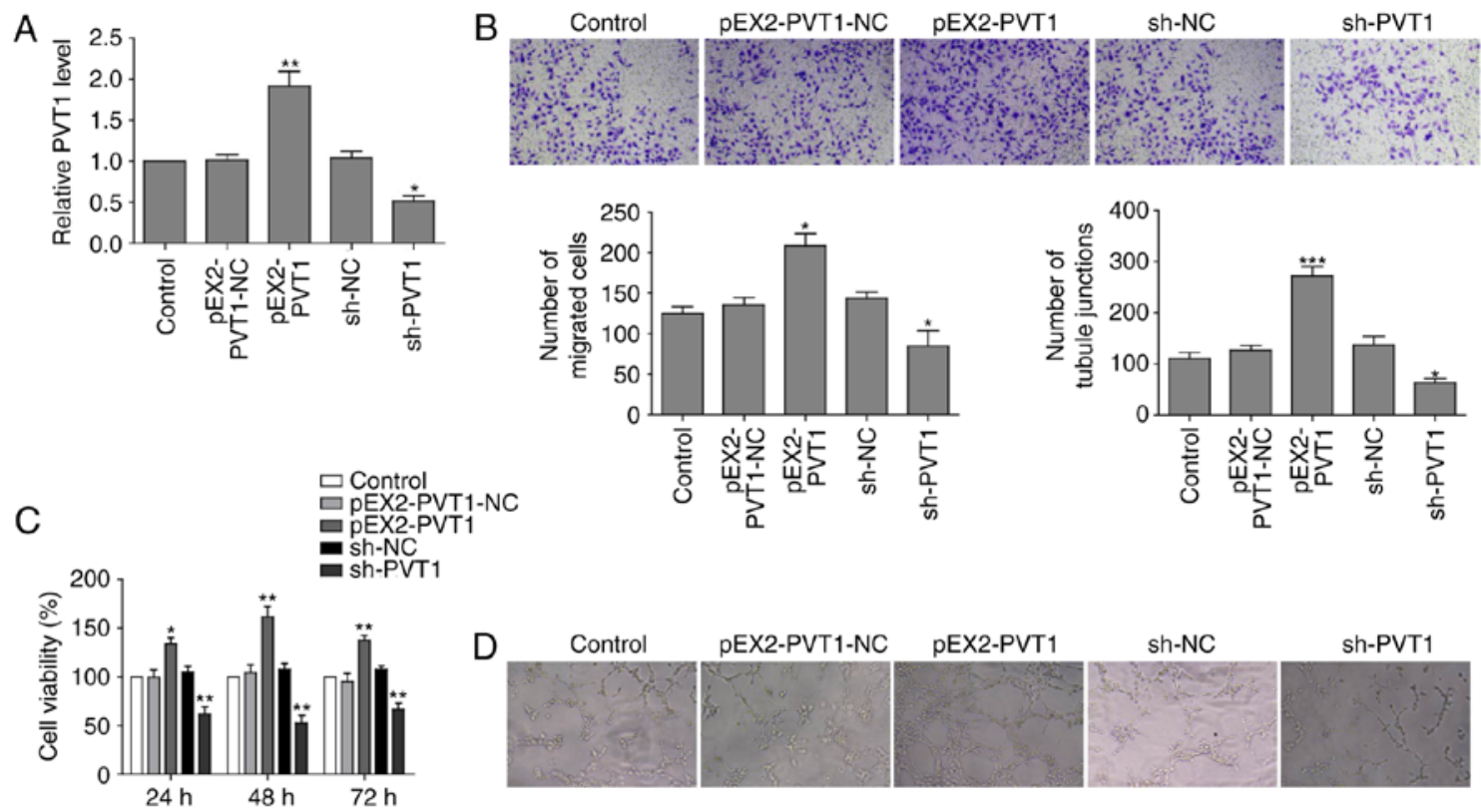

sh-NC

sh-PVT1

Figure 4. lncRNA PVT1 promotes cell proliferation, migration and in vitro tube formation of HUVECs cell. (A) Reverse transcription-quantitative polymerase chain reaction analysis of PVT1 expression level in HUVECs cells transfected with the indicated plasmids. GAPDH was used as internal control. ${ }^{*}<0.05$, ${ }^{* * *} \mathrm{P}<0.01$. vs. respective NC groups. (B) Transwell migration assay of HUVECs cells transfected with the indicated plasmids (upper) and statistical analysis of the migrated cells. ${ }^{*} \mathrm{P}<0.05$. vs. respective NC groups. (C) Viability assay of HUVECs cells transfected with the indicated plasmids by MTT assay. ${ }^{*} \mathrm{P}<0.05$ and ${ }^{* *} \mathrm{P}<0.01$. vs. respective NC groups (D) In vitro tube formation assay of HUVECs transfected with the indicated plasmids (upper) and statistical analysis of the tubule junctions. Data are presented as the mean \pm standard deviation. ${ }^{*} \mathrm{P}<0.05,{ }^{* * *} \mathrm{P}<0.001$ vs. respective NC groups. HUVEC, human umbilical vein endothelial cells; lncRNA, long noncoding RNA; PVT1, plasmacytoma variant translocation 1; sh, short hairpin RNA; NC, negative control. 

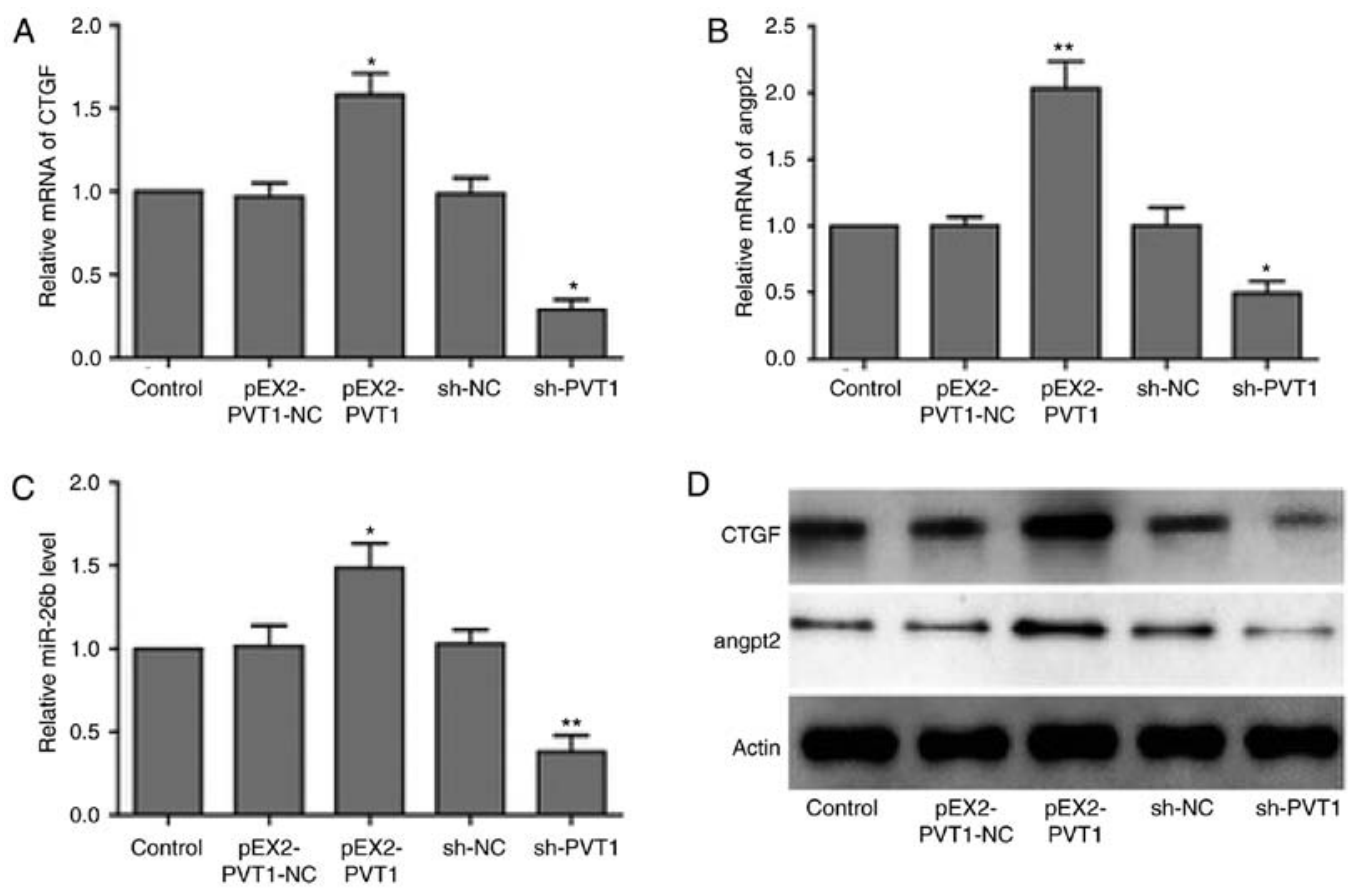

Figure 5. PVT1 suppresses expression of miR-26b, CTGF and ANGPT2. RT-qPCR analysis of (A) CTGF, (B) ANGPT2 and (C) miR-26b mRNA expression levels in HUVECs transfected with negative controls, PVT1 or shRNA against PVT1. GAPDH was used as internal control. " $\mathrm{P}<0.05$, ${ }^{* * *} \mathrm{P}<0.01 \mathrm{vs.}$ respective NC groups. (D) Western blot analysis of CTGF and ANGPT2 protein level in HUVECs cell transfected with negative controls, PVT1 or shRNA against PVT1. GAPDH was used as internal control. RT-qPCR, reverse transcription-quantitative polymerase chain reaction; shRNA, short hairpin RNA; CTGF, connective tissue growth factor; ANGPT2, angiopoietin 2; PVT1, plasmacytoma variant translocation 1; miR, microRNA; sh, short hairpin RNA; NC, negative control.
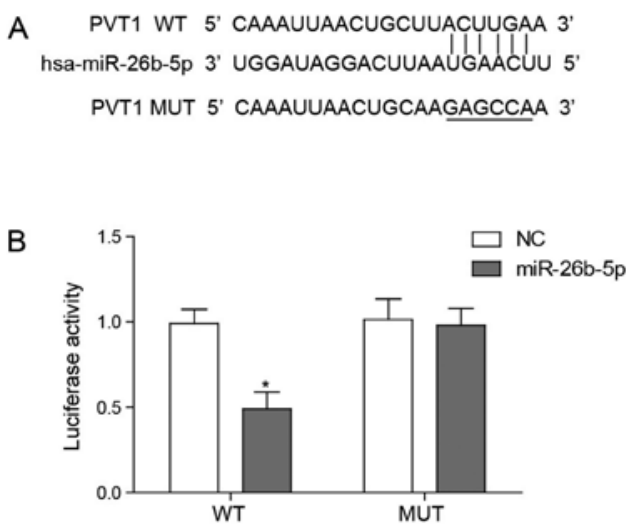

Figure 6. PVT1 directly interacts with miR-26b. (A) Bioinformatics analysis of match sequence of PVT1 within the sequence of miR-26b. PVT1-MUT denotes mutation of match sequence of PVT1. (B) Luciferase reporter assay indicates that WT-PVT1 binds miR-26b and not mutant PVT1. The relative luciferase activity was quantified and data are presented as the mean \pm standard deviation. "P $<0.05$ vs. respective NC groups. miR, microRNA; PVT1, plasmacytoma variant translocation 1; NC, negative control; WT, wild-type; MUT, mutant.

ANGPT2 protein expression levels in PVT1-overexpressed and PVT1-knockdown HUVECs (Fig. 5D). These findings suggested that PVT1 was able to bind to and subsequently downregulate miR-26b expression to promote the expression of CTGF and ANGPT2, facilitating angiogenesis of vascular endothelial cells.

PVT1 directly interacts with miR-26b. According to a previous study PVT1 has been identified to negatively regulate miR-26b expression, a previously reported regulator of angiogenesis (20). To confirm whether PVT1 regulated miR-26b through an interaction the present study used bioinformatics to predict the potential physical interaction between PVT1 and miR-26b (Fig. 6A). Subsequently, the present study conducted a dual luciferase reporter assay to confirm that PVT1 was able to bind directly and degrade miR-26b. It was demonstrated that only wild-type PVT1 significantly lowered the luciferase activity of miR-26b and the mutant form of PVT1 did not alter the luciferase activity (Fig. 6B).

\section{Discussion}

The present study demonstrated that PVT1 enhanced angiogenesis of vascular endothelial cells through binding miR-26b and subsequently increasing the expression of CTGF and ANGPT2. Bioinformatics and biochemical analyses demonstrated that PVT1 was able to directly bind to miR-26b and thus reduce the level of miR-26b. Subsequently, miR-26b regulation of two angiogenesis-promoting genes was investigated using RT-qPCR and western blot analysis. In addition, bioinformatics prediction and dual reporter luciferase assay revealed that miR-26b directly targeted the CTGF 3'-UTR. An MTT, Transwell assay and in vitro tube formation assays revealed that PVT1 promoted cell proliferation, migration and tube formation of HUVECs, respectively, whereas miR-26b exerted the opposite effects. Considered together, PVT1 had a stimulatory role in the angiogenesis of vascular endothelial cells by reducing the miR-26b expression level to increase CTGF and ANGPT2 expression.

Previous studies revealed that microRNAs regulate a variety of physiological and pathological processes, such as cardiovascular diseases. It has been reported that miR-143/145 are associated with vascular injury and hypertension and miR-499 is involved in myocardial ischemia (25-27). Additionally, miR-26a was identified to target the SMAD1-Id1-p2 ${ }^{\mathrm{WAF} / \mathrm{CIP} 1}$ 
signaling axis and inhibit angiogenesis in endothelial cells (18). However, the association between miR-26b and angiogenesis of vascular endothelial cells remains to be fully elucidated. To the best of our knowledge, the present study is the first to determine that miR-26b has an inhibitory role in angiogenesis of human endothelial cell through targeting CTGF and regulating its expression, which is consistent with previous studies that state that miR-26b inhibits tumorigenesis and metastasis of cancer cells partially by regulating CTGF $(28,29)$.

LncRNAs function to regulate gene expression by an epigenetic or post-transcriptional mechanism, including mRNA processing and degradation by interacting with a splicing factor and the 3'-UTR of the mRNA. Previous studies have reported that IncRNAs may act as sponges for microRNAs, which were coined as competing endogenous RNAs (ceRNAs) (30-32). These ceRNAs share sequences recognized by miRNAs termed microRNA recognition elements (MREs). Paci et al (33) developed a computational model to identify putative ceRNAs among lncRNAs in breast cancer. They determined that PVT1 had binding preference towards the miR-200 family, regulating the expression of hundreds of mRNAs. The present study verified that PVT1 was able to bind miR-26b via a complementary sequence. Therefore, PVT1 was important in angiogenesis of vascular endothelial cell as ceRNA to bind and degrade miR-26b, which expanded the current understanding of PVT1 as ceRNA to regulate biological processes.

In conclusion, the present study highlighted the importance of lncRNA PVT1 in promoting angiogenesis of vascular endothelial cells, which broadened the knowledge of how PVT1 has an effect in this process. It is of note that the findings of the present study provide the rationale for targeting PVT1 to treat angiogenesis abnormality-associated diseases, including cancer metastasis.

\section{Acknowledgements}

Not applicable.

\section{Funding}

This study was supported by the Natural Science Foundation of Jiangxi Province of China (grant no. 20151BBG70169).

\section{Availability of data and materials}

The datasets used and/or analyzed during the current study are available from the corresponding author on reasonable request.

\section{Authors' contributions}

JZ and LH conceived the study and guaranteed the integrity of the study; JZ, LH and ZY designed the study; JC defined intellectual content; JX performed research for the literature review, $\mathrm{ZZ}$ performed the experimental studies, YY acquired the data, JZ and LH performed statistical analysis, ZY prepared the manuscript and JZ and LH edited the manuscript.

\section{Ethics approval and consent to participate}

Not applicable.

\section{Consent for publication}

Not applicable.

\section{Competing interests}

The authors declare that there are no competing interests.

\section{References}

1. Wang KC and Chang HY: Molecular mechanisms of long noncoding RNAs. Mol Cell 43: 904-914, 2011.

2. Colombo T, Farina L, Macino G and Paci P: PVT1: A rising star among oncogenic long noncoding RNAs. BioMed Res Int 2015: 304208, 2015.

3. Yang YR, Zang SZ, Zhong CL, Li YX, Zhao SS and Feng XJ: Increased expression of the lncRNA PVT1 promotes tumorigenesis in non-small cell lung cancer. Int J Clin Exp Pathol 7: 6929-6935, 2014

4. Ding C, Yang Z, Lv Z, Du C, Xiao H, Peng C, Cheng S, Xie H, Zhou L, Wu J and Zheng S: Long non-coding RNA PVT1 is associated with tumor progression and predicts recurrence in hepatocellular carcinoma patients. Oncol Lett 9: 955-963, 2015.

5. Ding J, Li D, Gong M, Wang J, Huang X, Wu T and Wang C: Expression and clinical significance of the long non-coding RNA PVT1 in human gastric cancer. Onco Targets Ther 7: 1625-1630, 2014.

6. Zheng X, Hu H and Li S: High expression of lncRNA PVT1 promotes invasion by inducing epithelial-to-mesenchymal transition in esophageal cancer. Oncol Lett 12: 2357-2362, 2016.

7. Shen CJ, Cheng YM and Wang CL: LncRNA PVT1 epigenetically silences miR-195 and modulates EMT and chemoresistance in cervical cancer cells. J Drug Target 25: 1-8, 2017.

8. Chunharojrith P, Nakayama Y, Jiang X, Kery RE, Ma J, De La Hoz Ulloa CS, Zhang X, Zhou Y and Klibanski A: Tumor suppression by MEG3 lncRNA in a human pituitary tumor derived cell line. Mol Cell Endocrinol 416: 27-35, 2015.

9. Gordon FE, Nutt CL, Cheunsuchon P, Nakayama Y, Provencher KA, Rice KA, Zhou Y, Zhang X and Klibanski A: Increased expression of angiogenic genes in the brains of mouse meg3-null embryos. Endocrinology 151: 2443-2452, 2010.

10. Fu WM, Lu YF, Hu BG, Liang WC, Zhu X, Yang HD, Li G and Zhang JF: Long noncoding RNA Hotair mediated angiogenesis in nasopharyngeal carcinoma by direct and indirect signaling pathways. Oncotarget 7: 4712-4723, 2016.

11. Ma Y, Wang P, Xue Y, Qu C, Zheng J, Liu X, Ma J and Liu Y: PVT1 affects growth of glioma microvascular endothelial cells by negatively regulating miR-186. Tumour Biol 39: 1010428317694326, 2017.

12. Lewis BP, Burge CB and Bartel DP: Conserved seed pairing, often flanked by adenosines, indicates that thousands of human genes are microRNA targets. Cell 120: 15-20, 2005.

13. Yu J, Wang F, Yang GH, Wang FL, Ma YN, Du ZW and Zhang JW: Human microRNA clusters: Genomic organization and expression profile in leukemia cell lines. Biochem Biophys Res Commun 349: 59-68, 2006.

14. Bentwich I, Avniel A, Karov Y, Aharonov R, Gilad S, Barad O, Barzilai A, Einat P, Einav U, Meiri E, et al: Identification of hundreds of conserved and nonconserved human microRNAs. Nature Genet 37: 766-770, 2005.

15. Brennecke J, Hipfner DR, Stark A, Russell RB and Cohen SM: Bantam encodes a developmentally regulated microRNA that controls cell proliferation and regulates the proapoptotic gene hid in Drosophila. Cell 113: 25-36, 2003.

16. Calame K: MicroRNA-155 function in B Cells. Immunity 27: 825-827, 2007.

17. Jopling CL, Yi M, Lancaster AM, Lemon SM and Sarnow P: Modulation of hepatitis $C$ virus RNA abundance by a liver-specific MicroRNA. Science 309: 1577-1581, 2005.

18. Icli B, Dorbala P and Feinberg MW: An emerging role for the miR-26 family in cardiovascular disease. Trends Cardiovasc Med 24: 241-248, 2014.

19. Icli B, Wara AK, Moslehi J, Sun X, Plovie E, Cahill M, Marchini JF, Schissler A, Padera RF, Shi J, et al: MicroRNA-26a regulates pathological and physiological angiogenesis by targeting BMP/SMAD1 signaling. Circ Res 113: 1231-1241, 2013. 
20. Liu SC, Chuang SM, Hsu CJ, Tsai CH, Wang SW and Tang $\mathrm{CH}$ : CTGF increases vascular endothelial growth factor-dependent angiogenesis in human synovial fibroblasts by increasing miR-210 expression. Cell Death Dis 5: e1485, 2014.

21. Babic AM, Chen CC and Lau LF: Fisp12/mouse connective tissue growth factor mediates endothelial cell adhesion and migration through integrin alphavbeta3, promotes endothelial cell survival, and induces angiogenesis in vivo. Mol Cell Biol 19: 2958-2966, 1999.

22. Wang BJ, Ding HW and Ma GA: Long noncoding RNA PVT1 promotes melanoma progression via endogenous sponging miR-26b. Oncol Res, 2017.

23. Wang R, Ding X, Zhou S, Li M, Sun L, Xu X and Fei G: Microrna-26b attenuates monocrotaline-induced pulmonary vascular remodeling via targeting connective tissue growth factor (CTGF) and cyclin D1 (CCND1). Oncotarget 7: 72746-72757, 2016.

24. Livak KJ and Schmittgen TD: Analysis of relative gene expression data using real-time quantitative PCR and the 2(-Delta Delta C(T)) method. Methods 25: 402-408, 2001

25. Kuo CH, Chen PK, Chang BI, Sung MC, Shi CS, Lee JS Chang CF, Shi GY and Wu HL: The recombinant lectin-like domain of thrombomodulin inhibits angiogenesis through interaction with Lewis Y antigen. Blood 119: 1302-1313, 2012.

26. Boettger T, Beetz N, Kostin S, Schneider J, Kruger M, Hein L and Braun T: Acquisition of the contractile phenotype by murine arterial smooth muscle cells depends on the Mir143/145 gene cluster. J Clin Invest 119: 2634-2647, 2009.

27. Xin M, Small EM, Sutherland LB, Qi X, McAnally J, Plato CF, Richardson JA, Bassel-Duby R and Olson EN: MicroRNAs miR-143 and miR-145 modulate cytoskeletal dynamics and responsiveness of smooth muscle cells to injury. Genes Dev 23 2166-2178, 2009
28. Dorn GW 2nd, Matkovich SJ, Eschenbacher WH and Zhang Y: A human 3' miR-499 mutation alters cardiac mRNA targeting and function. Circ Res 110: 958-967, 2012.

29. Duan G, Ren C, Zhang Y and Feng S: MicroRNA-26b inhibits metastasis of osteosarcoma via targeting CTGF and Smad1. Tumour Biol 36: 6201-6209, 2015.

30. Guttman M, Amit I, Garber M, French C, Lin MF, Feldser D, Huarte M,Zuk O, Carey BW, Cassady JP, et al: Chromatin signature reveals over a thousand highly conserved large non-coding RNAs in mammals. Nature 458: 223-227, 2009.

31. KhalilAM,Guttman M,Huarte M,GarberM,RajA,RiveaMoralesD, Thomas K, Presser A, Bernstein BE, van Oudenaarden A, et al: Many human large intergenic noncoding RNAs associate with chromatin-modifying complexes and affect gene expression. Proc Natl Acad Sci USA 106: 11667-11672, 2009.

32. Huarte M, Guttman M, Feldser D, Garber M, Koziol MJ, Kenzelmann-Broz D, Khalil AM, Zuk O, Amit I, Rabani M, et al: A large intergenic noncoding RNA induced by p53 mediates global gene repression in the p53 response. Cell 142: 409-419, 2010.

33. Paci P, Colombo T and Farina L: Computational analysis identifies a sponge interaction network between long non-coding RNAs and messenger RNAs in human breast cancer. BMC Syst Biol 8: 83, 2014. 